\title{
Shelterin: the protein complex that shapes and safeguards human telomeres
}

\author{
Titia de Lange ${ }^{1}$ \\ The Rockefeller University, New York, New York 10021, USA
}

\begin{abstract}
Added by telomerase, arrays of TTAGGG repeats specify the ends of human chromosomes. A complex formed by six telomere-specific proteins associates with this sequence and protects chromosome ends. By analogy to other chromosomal protein complexes such as condensin and cohesin, I will refer to this complex as shelterin. Three shelterin subunits, TRF1, TRF2, and POT1 directly recognize TTAGGG repeats. They are interconnected by three additional shelterin proteins, TIN2, TPP1, and Rap1, forming a complex that allows cells to distinguish telomeres from sites of DNA damage. Without the protective activity of shelterin, telomeres are no longer hidden from the DNA damage surveillance and chromosome ends are inappropriately processed by DNA repair pathways. How does shelterin avert these events? The current data argue that shelterin is not a static structural component of the telomere. Instead, shelterin is emerging as a protein complex with DNA remodeling activity that acts together with several associated DNA repair factors to change the structure of the telomeric DNA, thereby protecting chromosome ends.
\end{abstract}

Six shelterin subunits: TRF1, TRF2, TIN2, Rap1, TPP1, and POT1

The components of shelterin were gradually identified over the past 10 years (Fig. 1). The first mammalian telomeric protein, now referred to as TRF1, was isolated based on its in vitro specificity for double-stranded TTAGGG repeats typical of vertebrate telomeres (Zhong et al. 1992; Chong et al. 1995). TRF2 was identified as a TRF1 paralog in the database (Bilaud et al. 1997; Broccoli et al. 1997) and TIN2 and Rap1 were found in two-hybrid screens with TRF1 and TRF2, respectively (Kim et al. 1999; Li et al. 2000). TPP1 (previously called TINT1 [Houghtaling et al. 2004], PTOP [Liu et al. 2004b], and PIP1 [Ye et al. 2004b]) recently emerged from searches for TIN2-interacting proteins. The most conserved component of shelterin, POT1, was identified based on se-

[Keywords: Shelterin; telomere; telomerase; cancer; DNA damage response]

${ }^{1}$ Correspondence.

E-MAIL delange@mail.rockefeller.edu; FAX (212) 327-7147.

Article and publication are at http://www.genesdev.org/cgi/doi/10.1101/ gad.1346005. quence homology to telomere end-binding factors in unicellular eukaryotes (Baumann and Cech 2001). Mass spectrometry on shelterin-associated factors failed to deliver additional components, suggesting that the tally of its subunits is nearing completion (Liu et al. 2004b; O'Connor et al. 2004; Ye et al. 2004a).

All six shelterin subunits can be found in a single complex in fractionated nuclear extracts (Liu et al. 2004a; Ye et al. 2004a). The linchpin of shelterin is TIN2, which tethers TPP1/POT1 to TRF1 and TRF2. TIN2 also connects TRF1 to TRF2 and this link contributes to the stabilization of TRF2 on telomeres (Liu et al. 2004a; Ye et al. 2004a). Shelterin subcomplexes containing either TRF1 or TRF2 in association with the other subunits can also be isolated. Although these subcomplexes could be an isolation artifact of the salt sensitivity of the TIN2TRF2 link (Ye et al. 2004a), photobleaching experiments also suggest that some of TRF1 and TRF2 are in separate complexes (Mattern et al. 2004). Further work is needed to establish the number of shelterin units bound per telomere, the stoichiometry of the shelterin subunits, and the significance of shelterin subcomplexes.

Not all proteins at chromosome ends are part of shelterin. Several criteria distinguish the shelterin components from the non-shelterin proteins observed at telomeres (Table 1). Shelterin is abundant at chromosome ends but does not accumulate elsewhere; it is present at telomeres throughout the cell cycle, and its known function is limited to telomeres. Non-shelterin proteins at chromosome ends fail to meet two or three of these criteria, yet can play important roles at telomeres.

Shelterin has exquisite specificity for telomeric TTAGGG repeats due to the presence of multiple TTAGGG recognition folds in the complex. The SANT/ Myb-type DNA-binding domains of TRF1 and TRF2 each bind the sequence $5^{\prime}$-YTAGGGTTR-3' in duplex DNA, showing very low tolerance for single-base changes (Fig. 1; Bianchi et al. 1999; Court et al. 2005; Hanaoka et al. 2005). TRF1 and TRF2 each form homodimers and higher order oligomers, so the collection of multiple DBDs they bring to the complex can peruse a large DNA sequence. POT1 also has strong sequence specificity, binding single-stranded $5^{\prime}$-(T)TAGGGT TAG-3' sites both at a $3^{\prime}$ end and at internal positions (Fig. 1; Lei et al. 2004; Loayza et al. 2004). Since these three shelterin subunits are connected through protein- 


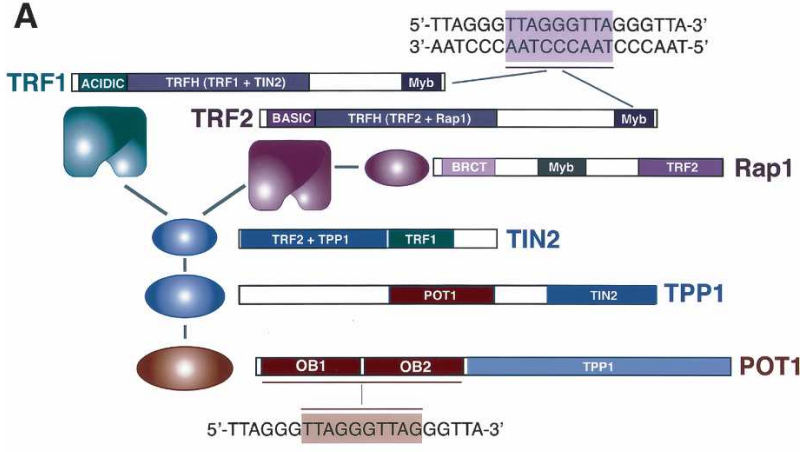

B
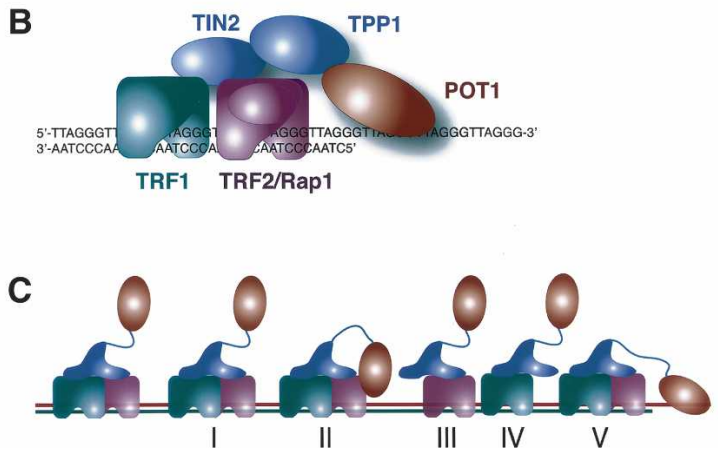

Figure 1. Shelterin. (A) The six known subunits of shelterin, their domain structure, protein interactions, and DNA-binding sites. POT 1 can bind its site both at a $3^{\prime}$ end and at an internal position (as shown). Not shown is the interaction between POT1 and TRF2 reported by Harris and colleagues (Yang et al. 2005). (B) Schematic of shelterin on telomeric DNA. For simplicity, POT1 is only shown as binding the site closest to the duplex telomeric DNA although it can also bind to the $3^{\prime}$ end. (C) Potential shelterin complexes and subcomplexes on telomeres. (I) Six-subunit shelterin with POT1 not bound to ssDNA. (II) As in I, with POT1 interacting with TRF2. (III) The TRF2/Rap1/TIN2/TPP1/POT1 complex. (IV) The TRF1/TIN2/ TPP1/POT1 complex. (V) The six-subunit shelterin with POT1 bound to single-stranded telomeric DNA. The flexible linker between the POT1 DBD and the rest of the shelterin complex is speculative. Nucleosomes have been omitted from this and all other schematics of the telomeric chromatin.

protein interactions, shelterin has the capacity to recognize telomeric DNA with at least five DNA-binding domains (two each in TRF1 and TRF2 and one in POT1). As a consequence, shelterin is uniquely qualified to distinguish telomeres from all other DNA ends.

Shelterin-related complexes are also found at telomeres in other eukaryotes. POT1-like proteins are present in nearly all eukaryotes (de Lange 2001), a TRF1/2 like protein is found in fission yeast and in trypanosomes (Cooper et al. 1998; Sfeir et al. 2005), and Rap1 is present in fungi (Shore 1994; Chikashige and Hiraoka 2001; Kanoh and Ishikawa 2001). In contrast, the shelterin subunits TIN2 and TPP1 have so far only been found in vertebrates; their emergence may have coincided with the gain of a second TRF-like gene. Thus, shelterin appears to be built up from a duplex telomeric DNA-bind- ing protein, a single-stranded DNA (ssDNA)-binding protein, and Rap1. The one exception to this rule may be Saccharomyces cerevisiae which lacks a TRF-like protein and uses instead a highly diverged Rap1 ortholog that binds double-stranded telomeric DNA. Conversely, yeast telomeres contain Rif1, a conserved protein that has no known role at mammalian telomeres and instead functions in the intra-S-phase checkpoint (Hardy et al. 1992; Silverman et al. 2004; Xu and Blackburn 2004). For these reasons, extrapolations from budding yeast to mammals can be specious as far as telomeres are involved.

\section{Shelterin shapes telomeres}

A major clue as to how shelterin protects telomeres comes from the observations that this complex affects the structure of telomeric DNA. At least three separate effects of shelterin have been documented. Shelterin determines the structure of the telomere terminus, it is implicated in the generation of t-loops, and it controls the synthesis of telomeric DNA by telomerase (Fig. 2).

A crucial way in which shelterin is thought to affect the structure of telomeric DNA is by forming t-loops (Fig. 2; Griffith et al. 1999; Stansel et al. 2001). Telomeres have a long single-stranded array of TTAGGG repeats at the 3' end (Makarov et al. 1997). This overhang has been proposed to invade the double-stranded telomeric DNA, base pairing with the C-strand and displacing the G-strand. The strand invasion takes place at a distance from the physical end of the telomeres and therefore results in a large duplex lariat structure, the t-loop (Fig. 2). The key feature of t-loops is that the end of the telomere is tucked in. The size of the circle part is probably not relevant since t-loops with very large (25$\mathrm{kb})$ and very small (1-kb) loops have been observed in human cells.

T-loops were first identified by electron microscopy of purified telomeric restriction fragments from human and mouse cells (Griffith et al. 1999). In order to observe t-loops in protein-free DNA, it is necessary to introduce interstrand cross-links with psoralen and UV. Without cross-links or proteins that stabilize the strand invasion, branch migration can dissociate them. Lariats have now also been observed in telomeric chromatin that was isolated without the use of psoralen, and in this analysis nucleosomes were found to be present on the loop as well as on the adjacent tail DNA (Nikitina and Woodcock 2004).

In vitro, shelterin components have DNA remodeling activities that are relevant to t-loop formation. TRF2 can remodel an artificial telomeric substrate into loops (Griffith et al. 1999; Stansel et al. 2001). These loops are stabilized by psoralen cross-linking, suggesting a strand-invasion event. The t-loop formation by purified TRF2 is puzzling since the reaction does not require ATP and TRF2 lacks a recognizable helicase domain. The reaction is not efficient, however, and it is likely that in vivo, TRF2 requires help of other factors to generate t-loops. 
Table 1. Examples of non-shelterin proteins at human telomeres

\begin{tabular}{|c|c|c|c|}
\hline Protein complex & Nontelomeric function & Effects at telomeres & Interactions \\
\hline Mre11/Rad50/Nbs1 & $\begin{array}{l}\text { recombinational repair } \\
\text { DNA damage sensor }\end{array}$ & $\begin{array}{l}\text { t-loop formation/resolution? } \\
\text { required for t-loop HR }\end{array}$ & associated with shelterin \\
\hline $\mathrm{ERCC} 1 / \mathrm{XPF}$ & $\begin{array}{l}\text { NER, crosslink repair } \\
3^{\prime} \text { flap endonuclease }\end{array}$ & $\begin{array}{l}\text { deficiency leads to formation of } \\
\text { TDMs; implicated in overhang } \\
\text { processing after TRF2 loss }\end{array}$ & associated with shelterin \\
\hline WRN helicase & $\begin{array}{l}\text { branch migration } \\
\text { G4 DNA resolution }\end{array}$ & $\begin{array}{l}\text { deficiency results in loss of lagging- } \\
\text { strand telomeres }\end{array}$ & TRF2 \\
\hline BLM helicases & $\begin{array}{l}\text { branch migration } \\
\text { crossover repression }\end{array}$ & t-loop formation/resolution? & TRF2 \\
\hline DNA-PK & NHEJ & $\begin{array}{l}\text { deficiency leads to mild } \\
\text { fusion phenotype }\end{array}$ & associated with shelterin \\
\hline PARP-2 & BER & not known & TRF2 \\
\hline Tankyrases & role in mitosis (tankyrase 1 ) & $\begin{array}{l}\text { positive regulator of telomere } \\
\text { length through inhibition of TRF1 }\end{array}$ & TRF1 \\
\hline Rad51D & unknown (HR?) & $\begin{array}{l}\text { deficiency leads to mild } \\
\text { fusion phenotype }\end{array}$ & unknown \\
\hline
\end{tabular}

Direct interactions with shelterin components are indicated where known. Factors recovered in association with shelterin are identified as such. Selected references: Mre11 complex, ERCC1/XPF, WRN, DNA-PK, see text. BLM (Yankiwski et al. 2000; Opresko et al. 2002; Stavropoulos et al. 2002); PARP-2 (Dantzer et al. 2004); Tankyrases (Smith et al. 1998; Smith and de Lange 2000; Kaminker et al. 2001; Chang et al. 2003; Dynek and Smith 2004; Ye and de Lange 2004); Rad51D (Tarsounas et al. 2004).

As shelterin associates with several proteins involved in recombinational repair (Table 1 ), it is anticipated that these factors could contribute to t-loop formation and maintenance (de Lange and Petrini 2000).

TRF1 also has DNA remodeling activity. In vitro, TRF1 can loop, bend, and pair telomeric repeat arrays, activities that might stimulate the folding of telomeres in vivo, (Bianchi et al. 1997, 1999; Griffith et al. 1998) and TIN2 can enhance some of TRF1's architectural effects (Kim et al. 2003). The DNA gymnastics of TRF1 are probably due to its unusually flexible binding mode. The two SANT/Myb domains of a TRF1 dimer reside at the end of flexible regions, explaining how they can engage their 5'-YTAGGGTTR-3' half-sites in different orientations and at variable distance. Now that the other components are largely known, it will be important to further define how shelterin remodels telomeric DNA in vitro and to test the contribution of the shelterin subunits to t-loop formation and maintenance in vivo.

T-loops are a conserved aspect of telomere structure and have been speculated to protect telomeres and regulate telomerase. Yet much about them remains to be determined. The exact structure at the base of the t-loop is not known and the role of TRF1 and TRF2 in t-loop formation has not (yet) been tested in vivo. It is also not clear whether t-loops are the only (or even the predominant) state of protected chromosome ends. Although the replication fork should dissociate the strand-invasion, it is not known whether DNA replication leads to a temporary "open" state. Addressing these questions is not simple because the detection of t-loops is currently constrained by the requirements of EM analysis.

Shelterin also affects the structure of the $3^{\prime}$ end. When either TRF2 or POT1 are inhibited, the overall amount of single-stranded TTAGGG repeats is diminished by 30\%-50\% (van Steensel et al. 1998; Hockemeyer et al.
2005). In the case of TRF2 inhibition, the loss of singlestranded TTAGGG DNA involves ERCC1/XPF, a flap endonuclease that can cleave next to a $3^{\prime}$ overhang just inside the neighboring duplex DNA (Zhu et al. 2003). The involvement of ERCC1/XPF predicts that some of the telomeres lose all of their ssDNA when TRF2 is inhibited. To address this issue, it will be necessary to apply techniques that measure changes in the length of the overhangs rather than loss of the overall singlestranded TTAGGG repeat signal. The protection of the $3^{\prime}$ overhang by shelterin could be an indirect effect of the formation of t-loops. For instance, the strand invasion of the $3^{\prime}$ overhang may be sufficient to protect the ssDNA from cleavage by ERCC1/XPF and other 3' flap nucleases. In addition, the binding of POT1 to the ssDNA could block nucleolytic degradation (Hockemeyer et al. 2005; Lei et al. 2005; Yang et al. 2005).

How is the $3^{\prime}$ overhang generated? Nuclease activity may be required to modify the telomere end generated by leading strand DNA synthesis, as its replication product may be a blunt end. Although the nuclease involved has not yet been identified, recent data shows that the nucleolytic processing of the 5' strand is controlled by shelterin. In a tour de force, Sfeir et al. (2005) were able to determine the sequence at the $3^{\prime}$ and $5^{\prime}$ ends of human chromosomes. They found that while the $3^{\prime}$ end is more or less randomly positioned within the TTAGGG repeats, the $5^{\prime}$ end is remarkably precise (Fig. 2A). Almost all human chromosomes have the sequence AATC CCAATC-5', indicating that the nucleolytic processing is regulated. The shelterin subunit POT1 is implicated in this control. When POT1 is inhibited, 5' ends lose their homogeneity and now end with AA, AT, TC, CC, CA, or AT (Hockemeyer et al. 2005).

A simple model for how POT1 controls the $5^{\prime}$ end sequence is suggested by its DNA-binding features (Fig. 2 ). In the natural structure of telomere, the first POT1 
A

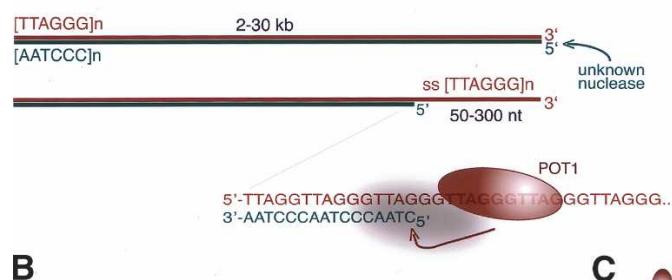

B
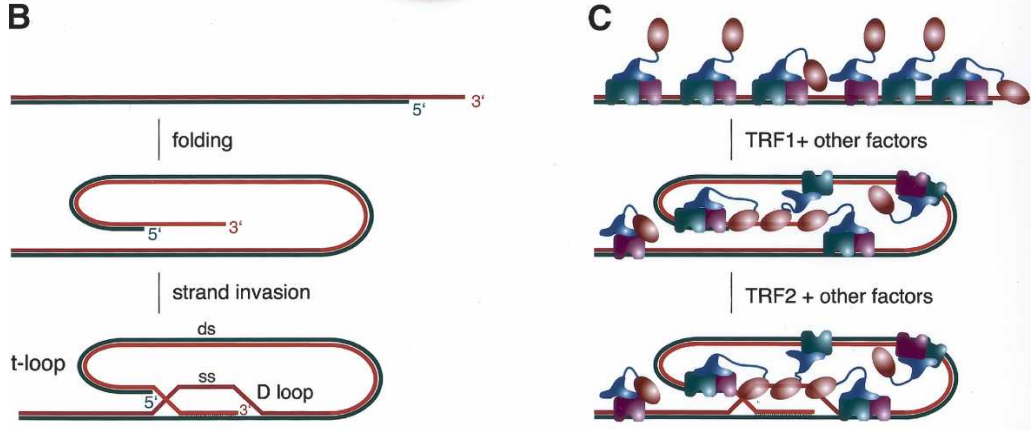

TRF2 + other factors

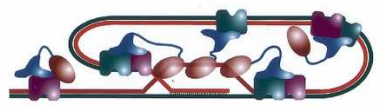

D
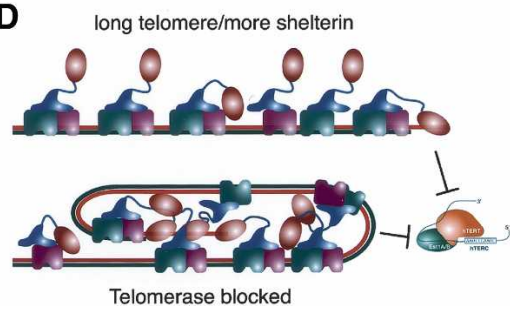

Telomerase blocked

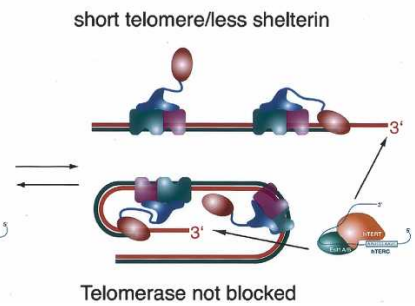

Figure 2. How shelterin may shape telomeres. $(A)$ Generation of the telomere terminus. After replication, chromosome ends require processing in order to acquire a long $3^{\prime}$ overhang. The nuclease involved is not known. The resulting $5^{\prime}$ end always has the sequence ATC- $5^{\prime}$. When POT1 is inhibited, this precision is lost. How POT1 determines the sequence of the $5^{\prime}$ end is not known, but the resulting terminal structure is a preferred binding site for POT1 in vitro. $(B)$ The t-loop structure. The 3 ' overhang is strand-invaded into the adjacent duplex telomeric repeat array, forming a D-loop. The size of the loop is variable. $(C)$ Speculative model for t-loop formation by shelterin. TRF1 has the ability to bend, loop, and pair telomeric DNA in vitro and could potentially fold the telomere. The shelterin component TRF2 can mediate t-loop formation in vitro. $(D)$ Model for telomere length regulation by shelterin. As the t-loop is unlikely to be a substrate for telomerase, telomeres are only shown in the "open" state (either in a linear or in a more compact folded configuration) that could be generated during $S$ phase. The presence of more shelterin on longer telomeres is proposed to increase the loading of POT1 on the telomeric overhang. POT1 bound to the $3^{\prime}$ end is proposed to block telomerase from acting. At the right, short telomeres with less shelterin are shown. Due to the diminished amount of shelterin, the chance that POT1 loads on the overhang is reduced, leading to a higher chance that telomerase can elongate the telomere. Forced increase of POT1 on telomeres (through shelterin overexpression) increases the chance that telomerase will be blocked, resulting in telomere shortening. Inhibition of shelterin, or a mutant of POT1 that does not bind ssDNA, reduces the chance that telomerase will be blocked, resulting in telomere elongation.

site in the $3^{\prime}$ overhang is just 2 nucleotides (nt) from the end of the duplex telomeric DNA. This configuration is a preferred binding substrate for POT1 in vitro (F. Ishikawa, pers. comm.; D. Hockemeyer and T. de Lange, unpubl.) suggesting that POT1 has an interaction with the duplex end of the telomere. So, once this terminal structure has been generated by a $5^{\prime}$ exonuclease, POT1 bound near the double-strand-single-strand transition may simply occlude the $5^{\prime}$ end from further processing. Obviously, the tethering of POT1 to the adjacent duplex telomeric DNA through the other shelterin proteins could further enhance the formation of a POT1 cap over the $5^{\prime}$ end.

A third way in which shelterin shapes telomeres is through its effect on telomere length maintenance (Fig. $2 D$ ). In yeast and mammals, telomeres are maintained within a set size range by a negative feedback loop that blocks the action of telomerase at individual chromosome ends (for review, see Smogorzewska and de Lange 2004). When a given telomere is too long, this cis-acting mechanism restrains the telomerase pathway. At a telomere that is too short, the control is relaxed so that telomerase can restore its length. Shelterin is a key component of this pathway, representing the cis-acting inhibitor. In the model, telomerase inhibition is a stochastic process influenced by the total amount of shelterin on a telomere. Since the amount of shelterin bound to a telomere is roughly proportional to the length of the TTAGGG repeat array, longer telomeres are proposed to have a greater probability of inhibiting telomerase. This control of telomerase requires the ssDNA-binding activity of the shelterin component POT1 (Loayza and de Lange 2003; Liu et al. 2004b). A POT1 mutant form that does not bind ssDNA results in complete loss of telomere length control. In this setting, telomerase generates very long telomeres that have lost the ability to inhibit the enzyme even though their shelterin load steadily increases (Loayza and de Lange 2003). It is easy to imagine how the ssDNA-binding activity of POT1 could block telomerase from gaining access to the 3 ' telomere terminus, and in vitro, such inhibitory activity has been noted (Kelleher et al. 2005; Lei et al. 2005). Based on these data, the current model is that the amount of shelterin on a telomere determines the likelihood that its POT1 component can position itself on the $3^{\prime}$ terminus and block telomerase (Fig. 2D).

\section{Shelterin inhibition activates the canonical DNA damage response}

It was long suspected that telomeres are "hidden" from the pathway that alerts cells to DNA damage. Evidence 
that dysfunctional human telomeres indeed activate the DNA damage response pathway first emerged from experiments in which the shelterin subunit TRF2 was inhibited with a dominant-negative allele (TRF2 $\left.{ }^{\Delta \mathrm{B} \Delta \mathrm{M}}\right)$, that heterodimerizes with the endogenous TRF2, blocking its binding to DNA (van Steensel et al. 1998; for review, see de Lange 2002). The loss of TRF2 activates the ATM kinase pathway, leading to p53 up-regulation and a p21-mediated G1/S arrest (Karlseder et al. 1999). Recent experiments in a mouse TRF2 knockout model have confirmed that TRF2 loss results in ATM activation and p53-dependent cell cycle arrest (Celli and de Lange 2005). The tumor suppressor p53 is also implicated in the response to telomere shortening. For instance, p53-deficient mice better tolerate the consequences of telomere shortening in the later generations of telomerase-deficient mice (Chin et al. 1999).

Telomere dysfunction can lead to either apoptosis or senescence. The outcome appears to be dictated by the cell type; fibroblasts undergo senescence upon TRF2 inhibition (and treatment with DNA damaging agents), whereas apoptosis is a more prominent outcome in lymphocytes and epithelial cells (Karlseder et al. 1999). The activation of the ATM pathway does not require the secondary DNA damage that can be generated when cells with dicentric chromosomes progress through mitosis. Rather, it is the damage at the telomere itself that activates the ATM kinase pathway (Fig. 3).

The view that deprotected telomeres activate the DNA damage response has been solidified by experiments in which DNA damage response factors were observed at telomeres (d'Adda di Fagagna et al. 2003; Takai et al. 2003). After inhibition of TRF2 or when telomeres become critically short, 53BP1, $\gamma$-H2AX, the Mre 11 complex, Rif1, and the phosphorylated form of ATM, ATM S1981-P, accumulate at chromosome ends. The cytological structures formed by the DNA damage factors are referred to as Telomere dysfunction Induced Foci (TIFs) (Takai et al. 2003). TIFs are also formed when other components of shelterin are inhibited (e.g., TIN2 or POT1) (Kim et al. 2004; Hockemeyer et al. 2005). ATMdeficient (A-T) cells have a decreased ability to form TIFs and the response is further reduced upon treatment with caffeine, an inhibitor of ATM, ATR, and other PI3 like kinases (PIKKs) (Takai et al. 2003). Based on these findings, it seems likely that both ATM and a second PIKK are responsible for the telomere damage response. ATR has been implicated as the second transducer by experiments on cells with shortened telomeres (Herbig et al. 2004). Simultaneous repression of ATM and ATR can reverse some of the phenotypes of telomere-directed senescence (d'Adda di Fagagna et al. 2003). Collectively, the data argue that dysfunctional telomeres are detected by the canonical DNA damage response. So far, there is no need to invoke a telomere "checkpoint" or specific signaling pathways to explain how cells respond to loss of telomere function.

Many aspects of the telomere damage response still need to be worked out. For instance, it is not known which sensors and mediators (e.g., the Mre11 complex,

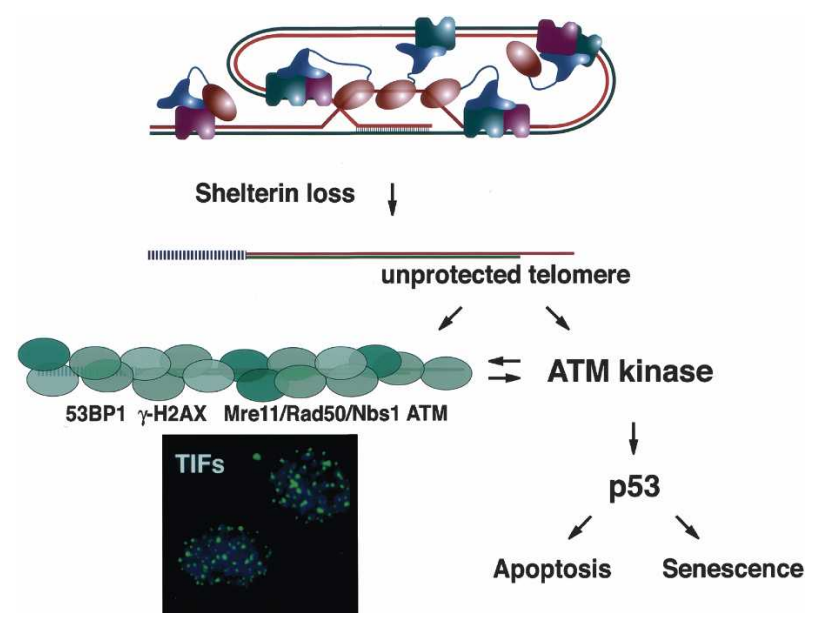

Figure 3. DNA damage response at dysfunctional telomeres. Telomeres lose protection after inhibition of shelterin subunits (e.g., TRF2, TIN2, or POT1) or telomere attrition. The molecular nature of the unprotected state is not known. Telomeres can become unprotected with or without overt change in the structure of the DNA (the latter is depicted). Upon loss of protection, telomeres become associated with DNA damage response factors forming the TIFs shown by the IF image of 53BP1 staining at telomeres. Telomere damage activates the ATM kinase, which leads to a p53-dependent G1/S arrest and can induce either apoptosis or senescence. In absence of ATM, the ATR kinase is thought to induce a cell cycle arrest. Telomere dysfunction also induces p16 (Jacobs and de Lange 2004), which can contribute to the inhibition of proliferation in p53-deficient human cells. In mouse cells, p16 is induced, but its induction does not affect entry into S phase (Smogorzewska and de Lange 2002).

the 9-1-1 complex, the Rad17 complex, RPA, 53BP1, MDC1) function in the telomere damage pathway, the relative contribution of ATM and ATR land perhaps other PIKKs) are not understood, and the nature of the telomere damage signal(s) has not been established.

\section{How does shelterin prevent a telomere damage signal?}

One possibility that has been considered is that the DNA damage response is activated when the $3^{\prime}$ overhang is lost. In agreement with this model, shelterin inhibition does indeed result in loss of (some of) the $3^{\prime}$ overhang DNA from mammalian telomeres. However, recent data indicate that this processing is not required for the DNA damage response. In DNA ligase IV-deficient mouse cells, TRF2 inhibition activates the ATM kinase and converts telomeres into DNA damage foci, even though the telomeric overhang remains intact (Celli and de Lange 2005).

If overhang protection is not sufficient to prevent the telomere damage response, shelterin must have at least one other mechanism to prevent detection of telomeres by the DNA damage surveillance. An interesting possibility is that t-loops create a nucleosomal organization that conceals the chromosome ends from the DNA dam- 
age surveillance. Recent work on ATM, 53BP1, and fission yeast Crb2 has suggested that a key event in the DNA damage response is a change in the nucleosomal organization at the site of DNA damage (Bakkenist and Kastan 2003; Huyen et al. 2004; Sanders et al. 2004). In the case of 53BP1, the critical interaction is with the dimethylated form of K79 of histone H3 (Huyen et al. 2004). This residue is constitutively methylated but may not be accessible in intact chromatin. The proposal is that when a DNA break occurs, the nucleosomal organization changes, exposing the binding site for $53 \mathrm{BP} 1$. With this mechanism in mind, it is easy to see how t-loops could hide the crucial nucleosome surface from the DNA damage surveillance. When a telomere is in the open state, the last nucleosome might have an exposed 53BP1 interaction site. In the t-loop, this penultimate nucleosome could be positioned such that the H3-K79 and other signaling residues are buried against other nucleosomes. However, 53BP1 is not the only (or the major) factor that senses DNA damage. The signal for other sensors, such as the Mre11 complex and 9-1-1/ RFC, have not been worked out. Once their sensing mechanisms are known, the counter-tactics of shelterin can be addressed. And vice versa, a better understanding of shelterin could provide a hint about how the DNA damage response detects damage at telomeres and elsewhere.

\section{An ATM inhibitor in shelterin?}

Unexpectedly, it was found that the shelterin subunit TRF2 has a weak interaction with the ATM kinase (Karlseder et al. 2004), although ATM is not detectable at telomeres. TRF2 binds to a region in ATM that contains Ser 1981, the residue that is autophosphorylated in response to DNA damage (Bakkenist and Kastan 2003). ATM autophosphorylation has been invoked as a key step in the activation of ATM, leading to the dissociation of kinase dimers that are thought to be less active than the monomers. In overexpression studies, TRF2 has the ability to inhibit S1981 phosphorylation and when TRF2 is abundant in the nucleoplasm, it has a dampening effect on the ATM signaling pathway (Karlseder et al. 2004). The proposal that shelterin may contain an ATM inhibitor is attractive because shelterin is abundant at telomeres but not elsewhere, so that it could restrain ATM at chromosome ends while not affecting the response to DNA damage elsewhere in the genome.

\section{Avoiding inappropriate repair}

As is the case for bulk DNA, telomeric DNA needs to be repaired. Base excision repair, nucleotide excision repair, and mismatch repair are presumably used to maintain the TTAGGG repeat sequence. But some forms of repair could have disastrous outcomes (Figs. 4, 5). Nonhomologous end-joining (NHEJ) of two telomeres creates a circular or a dicentric chromosome. Homologous recombination (HR) between telomeres could generate aberrant

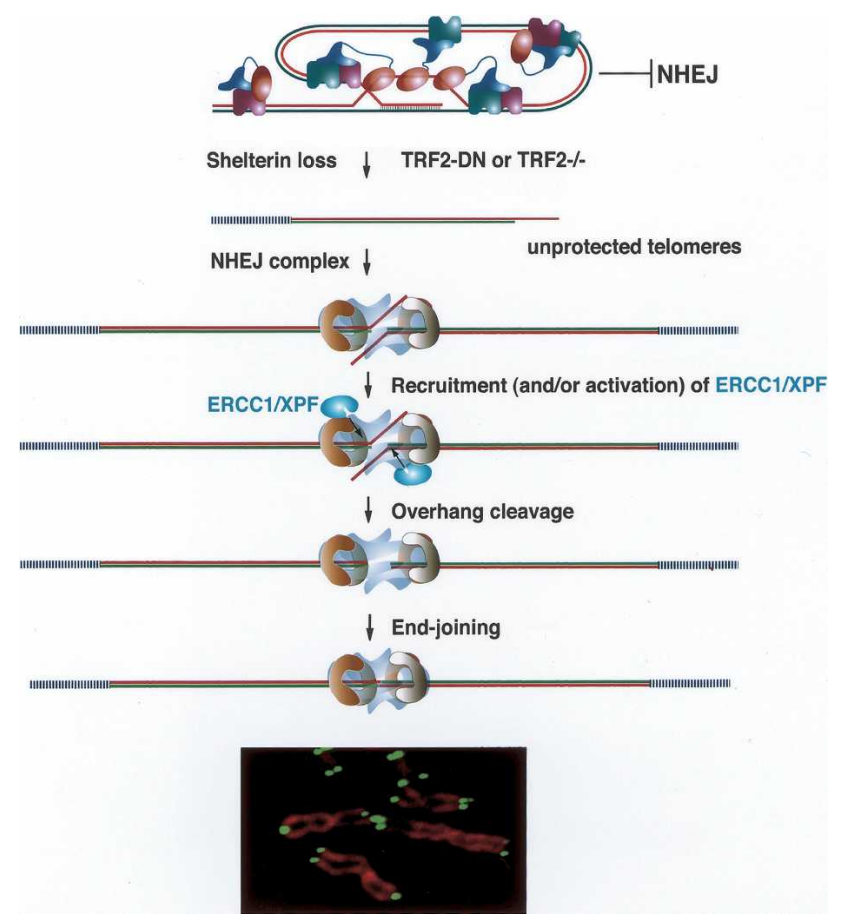

Figure 4. Proposed role for shelterin in protecting telomeres from NHEJ and overhang loss. Telomeres are proposed to be resistant to NHEJ because of their t-loop configuration, which will block the NHEJ complex from accessing to the chromosome end. Upon loss of shelterin, t-loops are proposed to be destabilized (or not formed), allowing engagement of the NHEJ pathway. Prior to the ligation of chromosome ends by DNA ligase IV, the DNA-PK complex is proposed to form a synaptic structure that activates and/or recruits ERCC1/XPF. This nuclease is implicated in cleavage of the $3^{\prime}$ overhang. End-joining of telomeres results in dicentric chromosomes (example shown at bottom). After DNA replication, fusions can occur between sister and non-sister telomeres. NHEJ can also occur prior to DNA replication, giving rise to chromosome-type fusions in metaphase (not shown).

telomere length and recombination of a telomere with interstitial telomeric sequence could lead to deletions, inversions, and translocations. Furthermore, homologous recombination threatens the integrity of the t-loop, potentially lopping off the loop. A new area of investigation focuses on establishing how shelterin prevents these events.

\section{Preventing NHEJ and overhang processing}

Chromosome end fusions are a prominent marker of telomere dysfunction. They occur when telomeres have become too short and when TRF2 is inhibited (for review, see de Lange 2002). These fusions are covalent connections between the C-strand of one telomere and the G-strand of another (Smogorzewska et al. 2002). They can occur before and after DNA replication and usually involve the ends of two different chromosomes. The resulting dicentric chromosomes can become attached to 


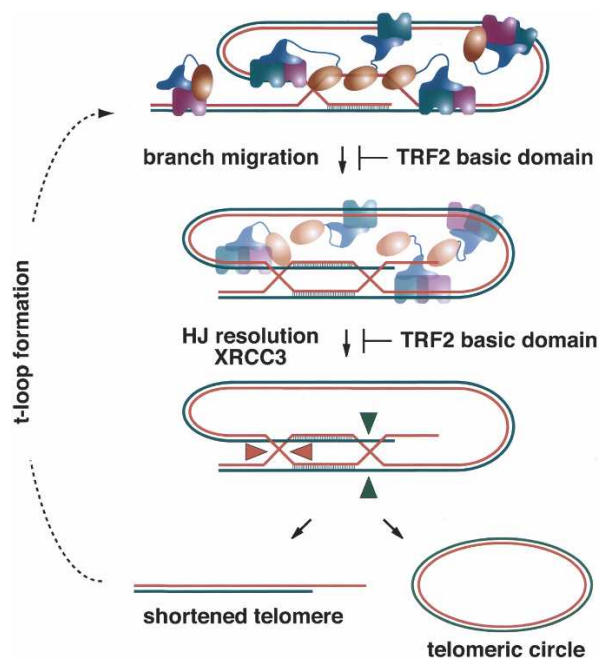

Figure 5. Control of t-loop HR by shelterin. Model depicting how late steps in HR can lead to sudden loss of telomeric DNA. Branch migration at the t-loop base can generate one or two HJs. Resolution of the double $\mathrm{HJ}$ in the direction shown will generate a shortened telomere and a circular telomeric DNA. T-loop $\mathrm{HR}$ is observed when an $\mathrm{N}$-terminal truncation mutant of TRF2, lacking the basic domain, is overexpressed. The circular product of t-loop HR is also observed in ALT cells and at very low levels in unperturbed normal human cells. In ALT cells, the circles could provide a mechanism for telomere maintenance through rolling-circle replication.

both spindle poles and lead to a problem for chromosome segregation in anaphase. Consequently, in anaphase cells with dicentric chromosomes, characteristic chromatin bridges are observed.

The fusion of damaged telomeres requires the same factors as normal NHEJ (Fig. 4; Smogorzewska et al. 2002; Celli and de Lange 2005; G. Celli, E. Denchi, and T. de Lange, unpubl.). Cells lacking DNA ligase IV or $\mathrm{Ku} 70$ have a 10- to 50 -fold reduced ability to fuse telomeres after shelterin inhibition. The other NHEJ proteins, DNA-PKcs, XRCC4, and Ku80, are presumed to be involved as well but have not been tested. The end-joining reaction of telomeres is an unusual type of NHEJ, since telomeres have a long 3 ' overhang. As mentioned above, the nucleotide excision repair factor ERCC1/XPF, an endonuclease with specificity for $3^{\prime}$ overhangs, has been implicated in the removal of the overhang (Zhu et al. 2003). As is the case with other end-trimming reactions, this step is dependent on the NHEJ machinery (Celli and de Lange 2005). How the NHEJ machinery activates (or recruits) ERCC1/XPF is not yet known.

One way in which shelterin could protect chromosome ends from NHEJ is by promoting t-loop formation. Without an accessible end, the NHEJ machinery will not be able to form the synaptic complex that is thought to be required for processing and ligation of the ends. By blocking access, t-loops would also protect the telomeric overhang from being removed by nucleases that depend on the NHEJ machinery (Fig. 4).

\section{Repression of HR}

Recently, it has become clear that telomeres also need to be protected from inappropriate homologous recombination. There are three types of HR that have detrimental outcomes at chromosome ends. The first is homologous recombination between sister telomeres, referred to as Telomere Sister Chromatid Exchange (T-SCE). T-SCEs could be detrimental to cells if the exchanged sequences are not equal in length. One sister telomere could become lengthened at the expense of the other. The exchange between sister telomeres can be detected by chromosome-orientation fluorescent in situ hybridization (CO-FISH) (for review, see Bailey et al. 2004). In the COFISH method, newly synthesized DNA is degraded and the remaining parental strands are detected with singlestranded probes. At telomeres, one parental strand is composed of 5'-TTAGGG-3' repeats and the other has only $5^{\prime}$-CCCTAA-3' sequences, allowing the two strands to be distinguished in metaphase chromosomes. After semiconservative replication, each chromatid in a metaphase chromosome will have a G-strand signal at one end and a C-strand signal at the other. A chromatid end displaying both C-strand and G-strand signals indicates that a T-SCE event has occurred at this end. In normal mouse and human cells, T-SCE is not frequent, but ALT cells, which maintain their telomeres by a recombination pathway, have very frequent T-SCE (Bailey et al. 2004; Bechter et al. 2004; Londono-Vallejo et al. 2004). The difference may be that T-SCEs are normally repressed and that ALT cells have loosened their control of HR at telomeres, allowing them to maintain their telomeres and therefore to survive (for review, see Neumann and Reddel 2002).

A second HR reaction that threatens telomeres is referred to as t-loop HR (Wang et al. 2004) (Fig. 5). T-loops are at risk for resolution by Holliday junction (HJ) resolvases because an $\mathrm{HJ}$ could be formed if the $5^{\prime}$ end of the telomere base pairs with the displacement loop (D loop). Branch migration in the direction of the centromere could generate a double $\mathrm{HJ}$ and resolution of this structure with crossover would delete the whole loop segment, leaving a drastically shortened telomere at the chromosome end. T-loop HR was discovered through a separation of function mutant of TRF2, TRF2 ${ }^{\triangle \mathrm{B}}$, which protects telomeres from NHEJ but induces sudden telomere truncations. These deletions are dependent on two proteins implicated in HR, the Mre11 recombination repair complex and XRCC3, a Rad51 paralog associated with $\mathrm{HJ}$ resolution activity. Cells expressing TRF2 ${ }^{\Delta \mathrm{B}}$ also contain extrachromosomal telomeric DNA that is circular. On two-dimensional gels, these circles show a broad size distribution consistent with their representing the loop part of the t-loops. How the $\mathrm{N}$ terminus of TRF2 represses t-loop HR has not been established. As unperturbed cells contain small amounts of circular telomeric DNA, suppression of the t-loop HR reaction at telomeres may be incomplete. The control of t-loop HR appears to be further relaxed in ALT cells, which contain abundant telomeric circles (Cesare and Griffith 2004; 
Wang et al. 2004). As T-SCE and t-loop HR are similar reactions (one taking place in cis, the other in trans), their prevalence in ALT cells may be due to loss of a repressor that controls both.

There may be a third type of HR with detrimental outcomes-the recombination between a telomere and interstitial telomeric DNA. Chromosome internal telomeric DNA is not frequent in human cells, but in many other vertebrates, such sequences are abundant throughout the chromosomes. Recombination between telomeres and these elements could generate terminal deletions, extrachromosomal fragments, inversions, and translocations. This type of recombination appears to take place in mouse cells lacking ERCC1, which generate large extrachromosomal elements that contain a single stretch of telomeric DNA, presumably at a chromosome internal site (Zhu et al. 2003). These elements, referred to as Telomeric DNA-containing Double Minute chromosomes (TDMs) could be formed by recombination between a telomere and interstitial telomeric DNA on the same chromosome. Perhaps shelterin carries ERCC1/XPF is on its "tool-belt" to prevent inappropriate recombination events.

\section{Shelterin's affiliations with DNA repair factors}

Shelterin has a startling number of interacting partners that function in DNA processing (Table 1). On the one hand, the factors on its "tool-belt" could facilitate shelterin tasks. On the other hand, these DNA-processing factors are potentially harmful to telomeres. This paradox suggests that shelterin must carefully control its affiliates.

A particularly perplexing case of telomere-associated DNA repair factors are DNA-PKcs and the Ku70/80 heterodimer (Hsu et al. 1999, 2000; d'Adda di Fagagna et al. 2001; O'Connor et al. 2004). These proteins promote NHEJ and are thought to associate with telomeres through interactions with shelterin (Table 1). How DNA-PKcs and $\mathrm{Ku}$ contribute to telomere function is not known; regardless, their behavior will have to be closely controlled so that NHEJ of chromosome ends is avoided. A similar conundrum is presented by the association of shelterin with ERCC1/XPF (Zhu et al. 2003), the nuclease implicated in the processing of the telomeric overhang after telomere damage. As suggested above, shelterin may be using this nuclease to block potentially harmful recombination between telomeres and interstitial telomeric DNA.

Another of shelterin's fair-weather friends is WRN, a RecQ helicase with 3' exonuclease activity. WRN interacts with TRF2 and can be detected at telomeres by ChIP and IF in S phase (Opresko et al. 2002, 2004; Crabbe et al. 2004; Machwe et al. 2004). The general function of WRN is to allow branch migration of HJs. In vitro, WRN can resolve the t-loop structure and degrade the 3 ' overhang, attributes that are potential threat to telomere integrity (Opresko et al. 2002, 2004). However, RecQ helicases also have the ability to remove G-quadruplex structures from TTAGGG repeats (Sun et al. 1998; Huber et al.
2002). This activity was recently proposed to be necessary for telomere replication based on the finding of Sphase-dependent telomere loss in the absence of WRN (Bai and Murnane 2003; Crabbe et al. 2004). WRN inhibition only affected lagging-strand DNA synthesis at telomeres, which copies the G-rich telomeric strand and is therefore expected to be inhibited by G-quadruplexes (Crabbe et al. 2004). So perhaps shelterin can use WRN to prevent problems during lagging-strand synthesis while blocking WRN from inappropriately resolving tloops after DNA replication is complete.

It is anticipated that the list of shelterin-affiliated factors will grow. The challenge will be to understand both the telomere-specific functions of these proteins and how shelterin manages to keep control over their unwanted activities.

\section{Conclusions}

The concept sketched here is that shelterin protects chromosome ends by actively changing the architecture of telomeric DNA. Shelterin recognizes telomeric DNA and remodels it into a t-loop, presumably so that the chromosome end is concealed from the NHEJ machinery. The shelterin subunit POT1 interacts with the single-stranded telomeric DNA, influencing the maintenance of telomeric DNA by telomerase and protecting the $5^{\prime}$ end of the chromosome. Yet other aspects of shelterin, so far largely unknown, allow telomeres to evade detection by the DNA damage surveillance. Shelterin appears to use a "tool-belt" containing several DNA repair proteins, such as WRN, ERCC1/XPF, the Mre11 complex, and DNA-PK, which are proposed to assist shelterin in executing additional DNA transactions that ensure the integrity of the chromosome end. At the same time, shelterin must control how these DNA repair factors act because some have the ability to destroy chromosome ends. Further deconstruction of the Byzantine world of telomeres should reveal how chromosome ends are protected and will provide a unique perspective on the DNA damage response.

\section{Acknowledgments}

Several important studies on human telomere function were not discussed in this review because of space constraints and thematic considerations. I apologize to colleagues whose work is not mentioned. I am very grateful to Tom Cech for helpful comments. Members of my lab are thanked for their critique of my ideas and writing style. We are grateful for the continuing support for our work of telomeres by grants from the NIH and the NCI (GM49046-12, CA76027-8, AG16642-7).

\section{References}

Bai, Y. and Murnane, J.P. 2003. Telomere instability in a human tumor cell line expressing a dominant-negative WRN protein. Hum. Genet. 113: 337-347.

Bailey, S.M., Brenneman, M.A., and Goodwin, E.H. 2004. Fre- 
quent recombination in telomeric DNA may extend the proliferative life of telomerase-negative cells. Nucleic Acids Res. 32: 3743-3751.

Bakkenist, C.J. and Kastan, M.B. 2003. DNA damage activates ATM through intermolecular autophosphorylation and dimer dissociation. Nature 421: 499-506.

Baumann, P. and Cech, T.R. 2001. Pot1, the putative telomere end-binding protein in fission yeast and humans. Science 292: $1171-1175$.

Bechter, O.E., Zou, Y., Walker, W., Wright, W.E., and Shay, J.W. 2004. Telomeric recombination in mismatch repair deficient human colon cancer cells after telomerase inhibition. Cancer Res. 64: 3444-3451.

Bianchi, A., Smith, S., Chong, L., Elias, P., and de Lange, T. 1997. TRF1 is a dimer and bends telomeric DNA. EMBO J. 16: $1785-1794$.

Bianchi, A., Stansel, R.M., Fairall, L., Griffith, J.D., Rhodes, D., and de Lange, T. 1999. TRF1 binds a bipartite telomeric site with extreme spatial flexibility. EMBO J. 18: 5735-5744.

Bilaud, T., Brun, C., Ancelin, K., Koering, C.E., Laroche, T., and Gilson, E. 1997. Telomeric localization of TRF2, a novel human telobox protein. Nat. Genet. 17: 236-239.

Broccoli, D., Smogorzewska, A., Chong, L., and de Lange, T. 1997. Human telomeres contain two distinct Myb-related proteins, TRF1 and TRF2. Nat. Genet. 17: 231-235.

Celli, G. and de Lange, T. 2005. DNA processing not required for ATM-mediated telomere damage response after TRF2 deletion. Nat. Cell. Biol. 7: 712-718.

Cesare, A.J. and Griffith, J.D. 2004. Telomeric DNA in ALT cells is characterized by free telomeric circles and heterogeneous t-loops. Mol. Cell. Biol. 24: 9948-9957.

Chang, W., Dynek, J.N., and Smith, S. 2003. TRF1 is degraded by ubiquitin-mediated proteolysis after release from telomeres. Genes \& Dev. 17: 1328-1333.

Chikashige, Y. and Hiraoka, Y. 2001. Telomere binding of the Rap1 protein is required for meiosis in fission yeast. Curr. Biol. 11: 1618-1623.

Chin, L., Artandi, S.E., Shen, Q., Tam, A., Lee, S.L., Gottlieb, G.J., Greider, C.W., and DePinho, R.A. 1999. p53 deficiency rescues the adverse effects of telomere loss and cooperates with telomere dysfunction to accelerate carcinogenesis. Cell 97: 527-538.

Chong, L., van Steensel, B., Broccoli, D., Erdjument-Bromage, H., Hanish, J., Tempst, P., and de Lange, T. 1995. A human telomeric protein. Science 270: 1663-1667.

Cooper, J.P., Watanabe, Y., and Nurse, P. 1998. Fission yeast Tazl protein is required for meiotic telomere clustering and recombination. Nature 392: 828-831.

Court, R., Chapman, L., Fairall, L., and Rhodes, D. 2005. How the human telomeric proteins TRF1 and TRF2 recognize telomeric DNA: A view from high-resolution crystal structures. EMBO Rep. 6: 39-45.

Crabbe, L., Verdun, R.E., Haggblom, C.I., and Karlseder, J. 2004. Defective telomere lagging strand synthesis in cells lacking WRN helicase activity. Science 306: 1951-1953.

d'Adda di Fagagna, F., Hande, M.P., Tong, W.M., Roth, D., Lansdorp, P.M., Wang, Z.Q., and Jackson, S.P. 2001. Effects of DNA nonhomologous end-joining factors on telomere length and chromosomal stability in mammalian cells. Curr. Biol. 11: 1192-1196.

d'Adda di Fagagna, F., Reaper, P.M., Clay-Farrace, L., Fiegler, H., Carr, P., Von Zglinicki, T., Saretzki, G., Carter, N.P., and Jackson, S.P. 2003. A DNA damage checkpoint response in telomere-initiated senescence. Nature 426: 194-198.

Dantzer, F., Giraud-Panis, M.J., Jaco, I., Ame, J.C., Schultz, I., Blasco, M., Koering, C.E., Gilson, E., Menissier-de Murcia, J., de Murcia, G., et al. 2004. Functional interaction between poly(ADP-Ribose) polymerase 2 (PARP-2) and TRF2: PARP activity negatively regulates TRF2. Mol. Cell. Biol. 24: 15951607.

de Lange, T. 2001. Telomere capping-one strand fits all. Science 292: 1075-1076.

. 2002. Protection of mammalian telomeres. Oncogene 21: $532-540$.

de Lange, T. and Petrini, J. 2000. A new connection at human telomeres: Association of the Mre11 complex with TRF2. Cold Spring Harb. Symp. Quant. Biol. LXV: 265-273.

Dynek, J.N. and Smith, S. 2004. Resolution of sister telomere association is required for progression through mitosis. Science 304: 97-100.

Griffith, J., Bianchi, A., and de Lange, T. 1998. TRF1 promotes parallel pairing of telomeric tracts in vitro. J. Mol. Biol. 278: 79-88.

Griffith, J.D., Comeau, L., Rosenfield, S., Stansel, R.M., Bianchi, A., Moss, H., and de Lange, T. 1999. Mammalian telomeres end in a large duplex loop. Cell 97: 503-514.

Hanaoka, S., Nagadoi, A., and Nishimura, Y. 2005. Comparison between TRF2 and TRF1 of their telomeric DNA-bound structures and DNA-binding activities. Protein Sci. 14: 119130.

Hardy, C.F., Sussel, L., and Shore, D. 1992. A RAP1-interacting protein involved in transcriptional silencing and telomere length regulation. Genes \& Dev. 6: 801-814.

Herbig, U., Jobling, W.A., Chen, B.P., Chen, D.J., and Sedivy, J.M. 2004. Telomere shortening triggers senescence of human cells through a pathway involving ATM, p53, and p21(CIP1), but not p16(INK4a). Mol. Cell 14: 501-513.

Hockemeyer, D., Sfeir, A.J., Shay, J.W., Wright, W.E., and de Lange, T. 2005. POT1 protects telomeres from a transient DNA damage response and determines how human chromosomes end. EMBO J. 20: 2667-2678.

Houghtaling, B.R., Cuttonaro, L., Chang, W., and Smith, S. 2004. A dynamic molecular link between the telomere length regulator TRF1 and the chromosome end protector TRF2. Curr. Biol. 14: 1621-1631.

Hsu, H.L., Gilley, D., Blackburn, E.H., and Chen, D.J. 1999. Ku is associated with the telomere in mammals. Proc. Natl. Acad. Sci. 96: 12454-12458.

Hsu, H.L., Gilley, D., Galande, S.A., Hande, M.P., Allen, B., Kim, S.H., Li, G.C., Campisi, J., Kohwi-Shigematsu, T., and Chen, D.J. 2000. Ku acts in a unique way at the mammalian telomere to prevent end joining. Genes \& Dev. 14: 28072812.

Huber, M.D., Lee, D.C., and Maizels, N.P. 2002. G4 DNA unwinding by BLM and Sgslp: Substrate specificity and substrate-specific inhibition. Nucleic Acids Res. 30: 3954-3961.

Huyen, Y., Zgheib, O., Ditullio Jr., R.A., Gorgoulis, V.G., Zacharatos, P., Petty, T.J., Sheston, E.A., Mellert, H.S., Stavridi, E.S., and Halazonetis, T.D. 2004. Methylated lysine 79 of histone $\mathrm{H} 3$ targets 53BP1 to DNA double-strand breaks. Nature 432: 406-411.

Jacobs, J.J. and de Lange, T. 2004. Significant role for p16(INK4a) in p53-Independent telomere-directed senescence. Curr. Biol. 14: 2302-2308.

Kaminker, P.G., Kim, S.H., Taylor, R.D., Zebarjadian, Y., Funk, W.D., Morin, G.B., Yaswen, P., and Campisi, J. 2001. TANK2, a new TRF1-associated PARP, causes rapid induction of cell death upon overexpression. J. Biol. Chem. 276: 35891-35899.

Kanoh, J. and Ishikawa, F. 2001. spRap1 and spRif1, recruited to telomeres by Taz1, are essential for telomere function in fission yeast. Curr. Biol. 11: 1624-1630. 
Karlseder, J., Broccoli, D., Dai, Y., Hardy, S., and de Lange, T. 1999. p53- and ATM-dependent apoptosis induced by telomeres lacking TRF2. Science 283: 1321-1325.

Karlseder, J., Hoke, K., Mirzoeva, O.K., Bakkenist, C., Kastan, M.B., Petrini, J.H., and de Lange, T. 2004. The telomeric protein TRF2 binds the ATM kinase and can inhibit the ATM-dependent DNA damage response. PLoS Biol. 2: E240.

Kelleher, C., Kurth, I., and Lingner, J. 2005. Human protection of telomeres 1 (POT1) is a negative regulator of telomerase activity in vitro. Mol. Cell. Biol. 25: 808-818.

Kim, S.H., Kaminker, P., and Campisi, J. 1999. TIN2, a new regulator of telomere length in human cells. Nat. Genet. 23: 405-412.

Kim, S.H., Han, S., You, Y.H., Chen, D.J., and Campisi, J. 2003. The human telomere-associated protein TIN2 stimulates interactions between telomeric DNA tracts in vitro. $E M B O$ Rep. 4: 685-691.

Kim, S.H., Beausejour, C., Davalos, A.R., Kaminker, P., Heo, S.J., and Campisi, J. 2004. TIN2 mediates functions of TRF2 at human telomeres. J. Biol. Chem. 279: 43799-43804.

Lei, M., Podell, E.R., and Cech, T.R. 2004. Structure of human POT1 bound to telomeric single-stranded DNA provides a model for chromosome end-protection. Nat. Struct. Mol. Biol. 11: 1223-1229.

Lei, M., Zaug, A.J., Podell, E.R., and Cech, T.R. 2005. Switching human telomerase on and off with hPOT1 protein in vitro. $J$. Biol. Chem. 280: 20449-20456.

Li, B., Oestreich, S., and de Lange, T. 2000. Identification of human Rap1: Implications for telomere evolution. Cell 101: 471-483.

Liu, D., O'Connor, M.S., Qin, J., and Songyang, Z. 2004a. Telosome, a mammalian telomere-associated complex formed by multiple telomeric proteins. J. Biol. Chem. 279:5133851342.

Liu, D., Safari, A., O'Connor, M.S., Chan, D.W., Laegeler, A., Qin, J., and Songyang, Z. 2004b. PTOP interacts with POT1 and regulates its localization to telomeres. Nat. Cell. Biol. 6: 673-680.

Loayza, D. and de Lange, T. 2003. POT1 as a terminal transducer of TRF1 telomere length control. Nature 424: 10131018.

Loayza, D., Parsons, H., Donigian, J., Hoke, K., and de Lange, T. 2004. DNA binding features of human POT1: A nonamer 5'-TAGGGTTAG-3' minimal binding site, sequence specificity, and internal binding to multimeric sites. J. Biol. Chem. 279: 13241-13248.

Londono-Vallejo, J.A., Der-Sarkissian, H., Cazes, L., Bacchetti, S., and Reddel, R.R. 2004. Alternative lengthening of telomeres is characterized by high rates of telomeric exchange. Cancer Res. 64: 2324-2327.

Machwe, A., Xiao, L., and Orren, D.K. 2004. TRF2 recruits the Werner syndrome (WRN) exonuclease for processing of telomeric DNA. Oncogene 23: 149-156.

Makarov, V.L., Hirose, Y., and Langmore, J.P. 1997. Long G tails at both ends of human chromosomes suggest a $\mathrm{C}$ strand degradation mechanism for telomere shortening. Cell 88: 657-666.

Mattern, K.A., Swiggers, S.J., Nigg, A.L., Lowenberg, B., Houtsmuller, A.B., and Zijlmans, J.M. 2004. Dynamics of protein binding to telomeres in living cells: Implications for telomere structure and function. Mol. Cell. Biol. 24: 5587-5594.

Neumann, A.A. and Reddel, R.R. 2002. Telomere maintenance and cancer-look, no telomerase. Nat. Rev. Cancer 2: 879-884.

Nikitina, T. and Woodcock, C.L. 2004. Closed chromatin loops at the ends of chromosomes. J. Cell. Biol. 166: 161-165.
O'Connor, M.S., Safari, A., Liu, D., Qin, J., and Songyang, Z. 2004. The human Rap1 protein complex and modulation of telomere length. J. Biol. Chem. 279: 28585-28591.

Opresko, P.L., Von Kobbe, C., Laine, J.P., Harrigan, J., Hickson, I.D., and Bohr, V.A. 2002. Telomere binding protein TRF2 binds to and stimulates the Werner and Bloom syndrome helicases. J. Biol. Chem. 277: 41110-41119.

Opresko, P.L., Otterlei, M., Graakjaer, J., Bruheim, P., Dawut, L., Kolvraa, S., May, A., Seidman, M.M., and Bohr, V.A. 2004. The Werner syndrome helicase and exonuclease cooperate to resolve telomeric $\mathrm{D}$ loops in a manner regulated by TRF1 and TRF2. Mol. Cell 14: 763-774.

Sanders, S.L., Portoso, M., Mata, J., Bahler, J., Allshire, R.C., and Kouzarides, T. 2004. Methylation of histone H4 lysine 20 controls recruitment of $\mathrm{Crb} 2$ to sites of DNA damage. Cell 119: 603-614.

Sfeir, A.J., Chai, W., Shay, J.W., and Wright, W.E. 2005. Telomere-end processing the terminal nucleotides of human chromosomes. Mol. Cell 18: 131-138.

Shore, D. 1994. RAP1: A protean regulator in yeast. Trends Genet. 10: 408-412.

Silverman, J., Takai, H., Buonomo, S.B., Eisenhaber, F., and de Lange, T. 2004. Human Rif1, ortholog of a yeast telomeric protein, is regulated by ATM and 53BP1 and functions in the S-phase checkpoint. Genes \& Dev. 18: 2108-2119.

Smith, S. and de Lange, T. 2000. Tankyrase promotes telomere elongation in human cells. Curr. Biol. 10: 1299-1302.

Smith, S., Giriat, I., Schmitt, A., and de Lange, T. 1998. Tankyrase, a poly(ADP-ribose) polymerase at human telomeres. Science 282: 1484-1487.

Smogorzewska, A. and de Lange, T. 2002. Different telomere damage signaling pathways in human and mouse cells. EMBO T. 21: 4338-4348.

. 2004. Regulation of telomerase by telomeric proteins. Annu. Rev. Biochem. 73: 177-208.

Smogorzewska, A., Karlseder, J., Holtgreve-Grez, H., Jauch, A., and de Lange, T. 2002. DNA ligase IV-dependent NHEJ of deprotected mammalian telomeres in G1 and G2. Curr. Biol. 12: 1635.

Stansel, R.M., de Lange, T., and Griffith, J.D. 2001. T-loop assembly in vitro involves binding of TRF2 near the 3' telomeric overhang. EMBO I. 20: E5532-E5540.

Stavropoulos, D.J., Bradshaw, P.S., Li, X., Pasic, I., Truong, K., Ikura, M., Ungrin, M., and Meyn, M.S. 2002. The Bloom syndrome helicase BLM interacts with TRF2 in ALT cells and promotes telomeric DNA synthesis. Hum. Mol. Genet. 11: 3135-3144.

Sun, H., Karow, J.K., Hickson, I.D., and Maizels, N.P. 1998. The Bloom's syndrome helicase unwinds G4 DNA. I. Biol. Chem. 273: 27587-27592.

Takai, H., Smogorzewska, A., and de Lange, T. 2003. DNA damage foci at dysfunctional telomeres. Curr. Biol. 13: 15491556.

Tarsounas, M., Munoz, P., Claas, A., Smiraldo, P.G., Pittman, D.L., Blasco, M.A., and West, S.C. 2004. Telomere maintenance requires the RAD51D recombination/repair protein. Cell 117: 337-347.

van Steensel, B., Smogorzewska, A., and de Lange, T. 1998. TRF2 protects human telomeres from end-to-end fusions. Cell 92: 401-413.

Wang, R.C., Smogorzewska, A., and de Lange, T. 2004. Homologous recombination generates T-loop-sized deletions at human telomeres. Cell 119: 355-368.

$\mathrm{Xu}$, L. and Blackburn, E.H. 2004. Human Rif1 protein binds aberrant telomeres and aligns along anaphase midzone microtubules. J. Cell. Biol. 167: 819-830. 
de Lange

Yang, Q., Zheng, Y.L., and Harris, C.C. 2005. POT1 and TRF2 cooperate to maintain telomeric integrity. Mol. Cell. Biol. 25: 1070-1080.

Yankiwski, V., Marciniak, R.A., Guarente, L., and Neff, N.F. 2000. Nuclear structure in normal and Bloom syndrome cells. Proc. Natl. Acad. Sci. 97: 5214-5219.

Ye, J.Z. and de Lange, T. 2004. TIN2 is a tankyrase 1 PARP modulator in the TRF1 telomere length control complex. Nat. Genet. 36: 618-623.

Ye, J.Z., Donigian, J.R., Van Overbeek, M., Loayza, D., Luo, Y., Krutchinsky, A.N., Chait, B.T., and de Lange, T. 2004a. TIN2 binds TRF1 and TRF2 simultaneously and stabilizes the TRF2 complex on telomeres. J. Biol. Chem. 279: 4726447271.

Ye, J.Z., Hockemeyer, D., Krutchinsky, A.N., Loayza, D., Hooper, S.M., Chait, B.T., and de Lange, T. 2004b. POT1interacting protein PIP1: A telomere length regulator that recruits POT1 to the TIN2/TRF1 complex. Genes \& Dev. 18: $1649-1654$.

Zhong, Z., Shiue, L., Kaplan, S., and de Lange, T. 1992. A mammalian factor that binds telomeric TTAGGG repeats in vitro. Mol. Cell. Biol. 12: 4834-4843.

Zhu, X.D., Niedernhofer, L., Kuster, B., Mann, M., Hoeijmakers, J.H., and de Lange, T. 2003. ERCC1/XPF removes the 3 ' overhang from uncapped telomeres and represses formation of telomeric DNA-containing double minute chromosomes. Mol. Cell 12: 1489-1498. 


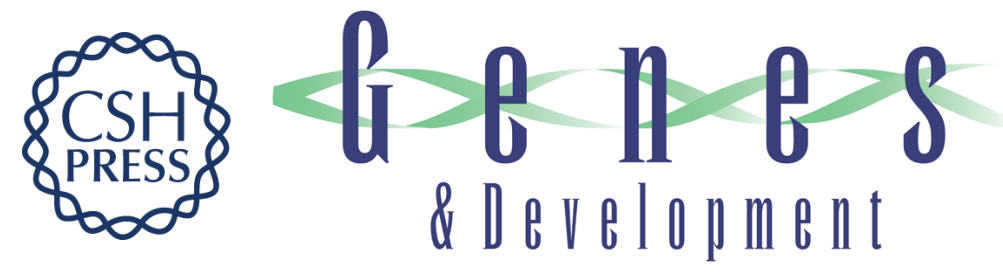

\section{Shelterin: the protein complex that shapes and safeguards human telomeres}

Titia de Lange

Genes Dev. 2005, 19:

Access the most recent version at doi:10.1101/gad.1346005

References This article cites 84 articles, 36 of which can be accessed free at: http://genesdev.cshlp.org/content/19/18/2100.full.html\#ref-list-1

License

Email Alerting Receive free email alerts when new articles cite this article - sign up in the box at the top Service right corner of the article or click here.

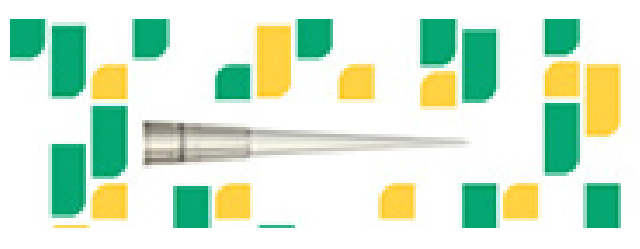

Focused on your science. 